\title{
Determining the Structural Basis of Cofactor and Substrate Interactions with Cdc48
}

Ian Cooney ${ }^{1}$, Han $\mathrm{Han}^{2}$, Chris Hill ${ }^{2}$ and Peter Shen ${ }^{2}$

${ }^{1}$ University of Utah, Midvale, Utah, United States, ${ }^{2}$ University of Utah, Salt Lake City, Utah, United States

Cdc48 (also known as p97 or VCP) is an essential and abundant AAA+ ATPase. It acts to separate proteins from a variety of contexts, including organelle membranes, ribosomes, protein complexes, and chromatin". This "segregase" activity is best described for unfolding proteins that are presented to the proteasome for degradation. Inhibition of human $\mathrm{Cdc} 48$ is an emerging therapeutic strategy for cancer treatment ${ }^{1}$ and mutations in the human homolog of $\mathrm{Cdc} 48$ are linked to diseases such as multisystem proteinopathy $^{2}$.

Cdc48 has a general structure of two ATPase domains capped by an N-terminal domain. Six Cdc48 subunits bind together in order to form a hexamer that has a central pore with pore loops from each subunit reaching into the pore. The $\mathrm{N}$-terminal domain that sits atop $\mathrm{Cdc} 48$ is responsible for the binding of most adaptor proteins of $\mathrm{Cdc} 48^{3}$. Until recently, all published structures show Cdc48 in a six-fold symmetric conformation with no substrate bound. This is true regardless of the nucleotide state of these structures 4 -5 However, these symmetrical structures most likely do not represent the active state of Cdc 48 .

The targeting of Cdc48 to substrates is directed by many adaptor proteins. Shp1 is an adaptor protein of $\mathrm{Cdc} 48$ that interacts with the $\mathrm{N}$-terminal domain. It is known to be important for cell cycle progression, and recent studies indicate this is due to direct interactions between Cdc48-Shp1 and protein phosphatase 1 (PP1) complexes ${ }^{6}$. By focusing on Shp1 as a substrate recruiting cofactor of Cdc48 recently we were able to visualize a $3.7 \AA$ resolution structure of Cdc48 actively translocating substrate ${ }^{7}$. Interestingly we were able to visualize Cdc48 using rapid purifications of endogenous Shp1 to then recover substrate bound Cdc48.

Our work and the investigation by other groups on related AAA+ ATPases has shown that the complexes form an asymmetric, spiral staircase structure in which five of the subunits wrap around the substrate in the central pore with one subunit disengaged ${ }^{7-8}$. These structures strongly suggest a hand-over-hand mechanism of substrate translocation in which ATP hydrolysis leads to subunit disengagement at the bottom of the staircase, while ATP binding leads to subunit reengagement at the top of the staircase ${ }^{7-8}$.

Interestingly, although the core hexamer of Cdc48 is generally well resolved in our structure, Shp1 which is the target of our purifications was unresolved, aside from one domain which we have been able to fit as bound to the N-terminal domain. By reprocessing our current data and collecting more data we have been able to resolve density sitting on top of $\mathrm{Cdc} 48$ that we had not previously observed (figure $1 \mathrm{~B}-\mathrm{C}$ ). This feature is in contact with the N-terminal domain and could likely belong to that of a cofactor bound to the N-terminal domain of Cdc48, such as Shp1. I am currently working towards resolving this density to a resolution that would allow us to accurately assign an identity to this density. 

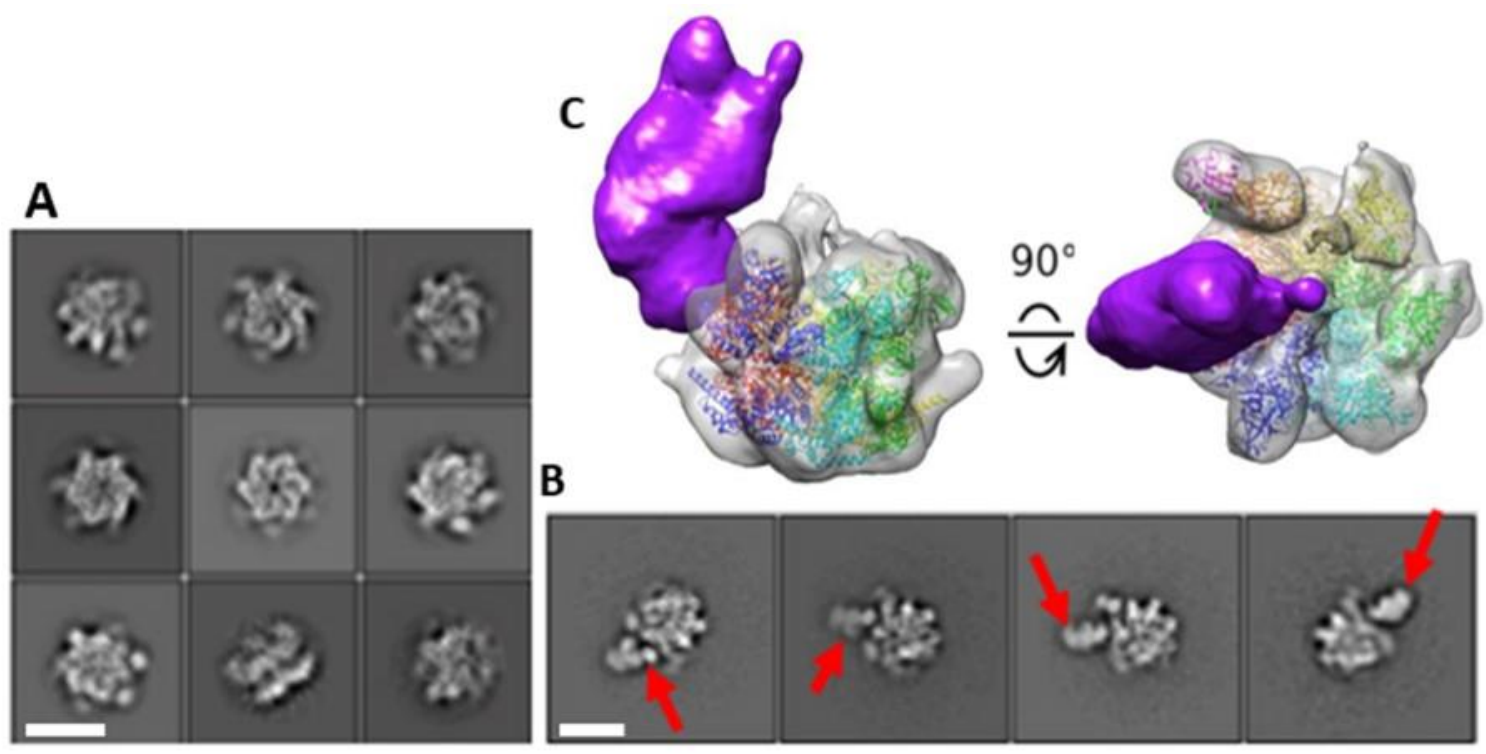

Figure 1. Resolving extra density bound to Cdc48(A) Representative 2D classifications for particles used in our refinement of the Cdc48 hexamer to $3.7 \AA$ but with no extra density apparent (7). Scale bar is 20 nm. (B) 2D classes with larger box size of representative classes possessing additional density (red arrows). Scale bar is $20 \mathrm{~nm}$. (C) Low resolution 3D reconstruction of Cdc48 with additional density, additional density including the $\mathrm{N}$-terminal domain of Subunit $\mathrm{A}$ is in purple whereas the rest of the core particle is in grey

\section{References}

1. Ye Y, Tang WK, Zhang T, Xia D. A Mighty "Protein Extractor" of the Cell: Structure and Function of the p97/CDC48 ATPase. Front Mol Biosci. 2017;4:39.

2. Wiehl CC, Dalal S, Pestronk A, Hanson PI. Inclusion body myopathy-associated mutations in p97/VCP impair endoplasmic reticulum-associated degradation. Human Molecular Genetics. 2006;15(2):189-99. Epub 2005/12/01.

3. Qiu L, Pashkova N, Walker JR, Winistorfer S, Allali-Hassani A, Akutsu M, Piper R, Dhe-Paganon S. Structure and function of the PLAA/Ufd3-p97/Cdc48 complex. J Biol Chem. 2010;285(1):365-72. doi: 10.1074/jbc.M109.044685. Epub 2009/11/02.

4. Banerjee S, Bartesaghi A, Merk A, Rao P, Bulfer SL, Yan Y, Green N, Mroczkowski B, Neitz RJ, Wipf P, Falconieri V, Deshaies RJ, Milne JL, Huryn D, Arkin M, Subramaniam S. A resolution cryo-EM structure of human p97 and mechanism of allosteric inhibition. Science. 2016;351(6275):871-5. Epub 2016/01/28.

5. Bodnar NO, Kim KH, Ji Z, Wales TE, Svetlov V, Nudler E, Engen JR, Walz T, Rapoport TA. Structure of the Cdc48 ATPase with its ubiquitin-binding cofactor Ufd1-Np14. Nature Structural Molecular Biology. 2018;25(7):616-622. Epub 2018/07/02.

6. Cheng YL, Chen RH. Assembly and quality control of the protein phosphatase 1 holoenzyme involves the Cdc48-Shp1 chaperone. Journal of Cell Science. 2015;128(6):1180-92. Epub 2015/01/22.

7. Cooney, I., Han, H., Stewart, M.G., Carson, R.H., Hansen, D.T., Iwasa, J.H., Price, J.C., Hill, C.P. and Shen, P.S. Structure of the Cdc48 segregase in the act of unfolding an authentic substrate. Science. 2019;365(6452):502-505. Epub 2019/06/27. doi: 10.1126/science.aax0486

8. Han H, Monroe N, Sundquist WI, Shen PS, Hill CP. The AAA ATPase Vps4 binds ESCRT-III substrates through a repeating array of dipeptide-binding pockets. Elife. 2017;6:e31324. 